
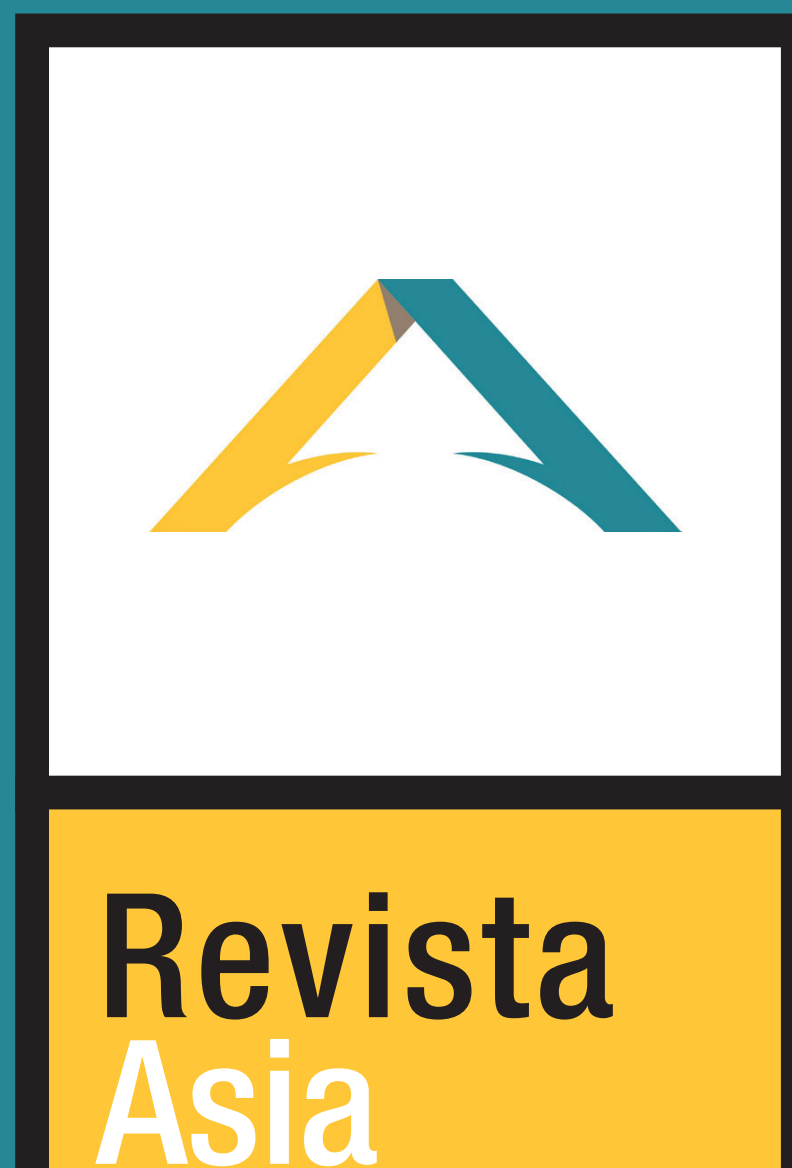

América Latina

Año 1. Volumen 1. Número 1. JUNIO 2016. Argentina ISSN 2524-9347

Grupo de Estudios de Asia y América Latina Instituto de Estudios sobre América Latina y el Caribe Universidad de Buenos Aires

\section{PRESENTACIÓN}

¿POR QUÉ UNA REVISTA SOBRE ASIA Y

AMERICA LATINA?

Fernando Pedrosa 5

\section{DOSSIER}

EL AMOR EN DOS LEYENDAS DEL SUDESTE ASIÁTICO

Mireya Sosa Abella 13

LA POLÍTICA EXTERIOR DE ARGENTINA HACIA TAILANDIA: CONDICIONAMIENTOS POLÍTICOS Y ECONÓMICOS DENTRO DE UNA ESTRATEGIA DE DIVERSIFICACIÓN
M. Florencia Rubiolo
29

INTERNACIONALIZACIÓN Y DIPLOMACIA GASTRONÓMICA EN TAILANDIA

Ezequiel Ramoneda 49

BREVE ANÁLISIS COMPARATIVO DE LA CAPOEIRA BRASILERA Y LA MÚSICA SAMARA TAILANDESA Y LAS PRÁCTICAS RITUALES COMPARTIDAS

Duncan Williams

67

\section{VARIA}

LOS DESAFÍOS POLÍTICOS EN CHINA HOY

Cristina Reigadas

87

\section{DIÁLOGOS}

¿QUÉ HACEMOS CON LOS VIEJOS? UNA SIMPLE PREGUNTA NO TIENE POR QUÉ TENER SIMPLE RESPUESTA: CHINA

Diego Bernardini 105 


\section{Dirección}

Dr. Fernando Pedrosa

(Grupo de Estudios de Asia y América Latina, Instituto de Estudios sobre América Latina y el Caribe, Universidad de Buenos Aires, Argentina)

\section{Secretaría de Redacción}

\section{Mg. Cecilia Noce}

(Grupo de Estudios de Asia y América Latina, Instituto de Estudios sobre América Latina y el Caribe, Universidad de Buenos Aires, Argentina)

\section{Dr. Ariel Sribman}

(Universidad de Girona, España)

\section{Consejo Editorial}

\section{Dra. Mercedes Botto}

(FLACSO-Argentina)

Dra. María José Bruña

(Universidad de Salamanca, España)

Dra. Pasuree Luesakul

(Directora del Centro de Estudios Latinoamericanos, Universidad de Chulalongkorn, Tailandia).

\section{Lic. Patricia Piccolini}

(Directora Carrera de Edición, Facultad de Filosofía y Letras, Universidad de Buenos Aires, Argentina).

\section{Lic. Ezequiel Ramoneda}

(Centro de Estudios del Sudeste asiático. Instituto de Relaciones Internacionales Universidad Nacional de La Plata, Argentina).

Dra. Cristina Reigadas

(Instituto Gino Germani, Facultad de Ciencias Sociales, Universidad de Buenos Aires, Argentina)

Dra. Florencia Rubiolo

(Universidad Nacional de Córdoba, Argentina)

Dra. Mireya Sosa Abella

(Universidad de Malasia)

Dr. Jaime Moreno Tejada

(Universidad de Chulalongkorn, Tailandia)

Dr. Ignacio Tredici

(ex Jefe del Equipo Jurídico de la Oficina del Co-Juez de Instrucción Internacional del Tribunal Khmer Rojo, United Nations Mission of Assistance to the Khmer Rouge Trials, Extraordinary Chambers in the Courts of Cambodia)

Dr. Wasana Wongsurabat

(Universidad de Chulalongkorn, Tailandia)

\section{Eudeba}

Universidad de Buenos Aires

$1^{\circ}$ edición: junio 2016

(C) 2016

Editorial Universitaria de Buenos Aires

Sociedad de Economía Mixta

Av. Rivadavia 1571/73 (1033) Ciudad de Buenos Aires

Tel: 4383-8025 / Fax: 4383-2202

www.eudeba.com.ar

DG: Alessandrini \& Salzman para Eudeba.

Impreso en Argentina

Hecho el depósito que establece la ley 11.723

No se permite la reproducción total o parcial de este libro, ni su almacenamiento en un sistema informático, ni su transmisión en cualquier forma o por cualquier medio, electrónico, mecánico, fotocopia u otros métodos, sin el permiso previo del editor. 


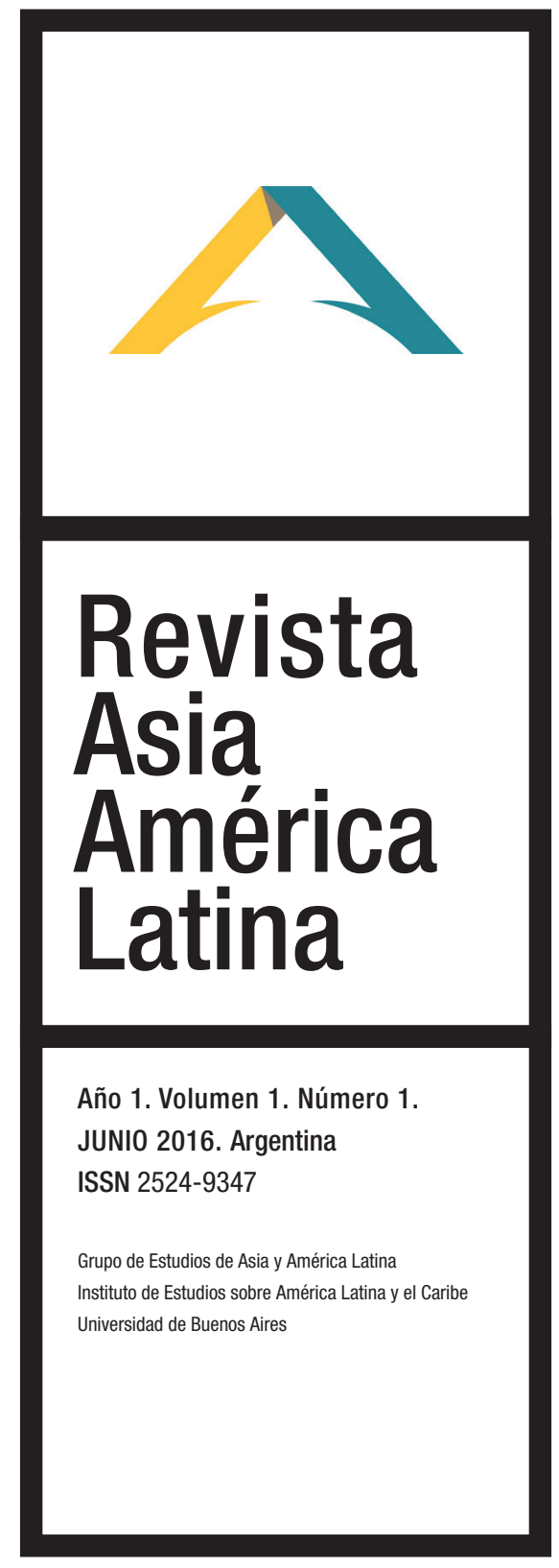

\section{PRESENTACIÓN}

¿POR QUÉ UNA REVISTA SOBRE ASIA Y AMERICA

LATINA?

Fernando Pedrosa 5

\section{DOSSIER}

EL AMOR EN DOS LEYENDAS DEL SUDESTE

ASIÁTICO

Mireya Sosa Abella 13

LA POLÍTICA EXTERIOR DE ARGENTINA HACIA TAILANDIA: CONDICIONAMIENTOS POLÍTICOS Y ECONÓMICOS DENTRO DE UNA ESTRATEGIA DE DIVERSIFICACIÓN

M. Florencia Rubiolo 29

INTERNACIONALIZACIÓN Y DIPLOMACIA GASTRONÓMICA EN TAILANDIA

Ezequiel Ramoneda 49

BREVE ANÁLISIS COMPARATIVO DE LA CAPOEIRA BRASILERA Y LA MÚSICA SAMARA TAILANDESA Y LAS PRÁCTICAS RITUALES COMPARTIDAS

Duncan Williams 67

\section{VARIA}

LOS DESAFÍOS POLÍTICOS EN CHINA HOY

Cristina Reigadas 87

\section{DIÁLOGOS}

¿QUÉ HACEMOS CON LOS VIEJOS? UNA SIMPLE PREGUNTA NO TIENE POR QUÉ TENER SIMPLE RESPUESTA: CHINA

Diego Bernardini 105

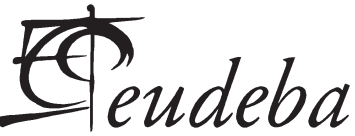





\title{
LA POLÍTICA EXTERIOR DE \\ ARGENTINA HACIA TAILANDIA: \\ CONDICIONAMIENTOS POLITICOS \\ Y ECONÓMICOS DENTRO DE UNA \\ ESTRATEGIA DE DIVERSIFICACIÓN
}

\author{
ARGENTINA'S FOREIGN POLICY \\ TOWARD THAILAND: POLITICAL AND \\ ECONOMICAL CONSTRAINS WITHIN A \\ DIVERSIFICATION STRATEGY.
}

\author{
M. Florencia Rubiolo \\ CIECS (CONICET y UNC) / USiglo21 \\ frubiolo@gmail.com
}

RESUMEN: La política exterior Argentina y su modo de inserción internacional han atravesado algunos cambios en la última década. La mayor parte de ellos estuvo articulada con modificaciones en la orientación económica comercial y en el modelo de desarrollo interno. La vinculación del país con el Sudeste de Asia se enmarca en una estrategia de diversificación comercial -y en parte política- que se inscribe en los nuevos lineamientos externos. Uno de los principales socios de Argentina en la región entre 2003-2015 fue Tailandia. Aunque la relación comercial bilateral mantuvo un recorrido incremental sostenido, la relación político-diplomática se diferenció del resto de la región asiática porque atravesó períodos de mayor actividad y acercamiento, y de enfriamiento respondiendo a condiciones internas y externas a la Argentina. En este trabajo nos proponemos abordar este vínculo bilateral tomando como variables centrales para comprender esta vinculación la relación con la orientación económica interna, y también con variables de corte político-institucional e ideológico que tuvieron, hacia finales del período, un mayor peso en el devenir de las relaciones bilaterales.

Palabras clave: política exterior argentina - inserción - diversificación - Tailandia

ABSTRACT: Argentina's foreign policy and its mode of international integration have gone through some changes over the last decade. Most of them were articulated with changes in trade and economic orientation in the model of internal development. Linking the country with Southeast Asia is part of a strategy of commercial and political diversification that is framed by the new external guidelines. One of the main partners of Argentina in the region between 2003- 
2015 was Thailand. Although the bilateral trade relationship maintained a sustained incremental travel, the political- diplomatic relationship differed from the rest of the Asian region because it went through periods of higher activity and approach, and of cooling of relations that responded to Argentina's internal and external conditions. In this paper we propose to address this bilateral link taking as central variables to understanding this relationship the articulation with the domestic economic orientation, and also with varying political - institutional and ideological conditions that, by the end of the period, had a greater impact in the evolution of bilateral relations.

Keywords: Argentina's foreign policy - insertion - diversitication - Thailand

\section{Introducción}

La política exterior y la inserción económica-comercial de la Argentina han mostrado históricamente una estrecha articulación con los objetivos económicos internos. Esto se debe a que, como ha sido afirmado por numerosos autores, el modo de inserción externa y las acciones de política exterior se delinean en función del modelo económico nacional (Van Klaveren, 1992; Busso, 2014). De esta manera, ante diferentes definiciones desde el plano interno, resltan diversas orientaciones de política exterior -en sus varias dimensiones- así como una cambiante definición de los socios prioritarios.

Esta articulación directa entre modelo económico interno y política exterior no está exenta de excepciones, es decir, de acciones que no se inscriben en la lógica interna de la relación. Sin embargo, nuestro punto aquí es sentar esta vinculación como la premisa de partida de nuestro trabajo, sin la cual el análisis de la política exterior argentina hacia cualquier Estado u organismo externo resulta incompleta. Esto implica que la orientación de la política económica del gobierno se convierte en un condicionante de primer orden en la política exterior y el modo de inserción económica internacional de un Estado como la Argentina.

Los condicionamientos internos, no obstante, no se limitan solo a la orientación económica nacional, existen también condicionantes de naturaleza institucional, ideológica, científica, cultural, geográfica y estratégica, que afectan la política exterior. A estas variables se suman también algunas de naturaleza externa, que tienen, dependiendo del momento, diferente impacto en la política exterior del país ${ }^{1}$. Aunque en menor medida que el modelo económico, estas condiciones tienen asimismo incidencia en la definición de los socios prioritarios y las acciones de política externa.

1. Alberto Van Klaveren (1992) realiza una clasificación de las condiciones que inciden en las decisiones de política exterior de los países latinoamericanos, distinguiendo entre condicionantes internos y externos, y el campo temático. 
En este trabajo nos proponemos abordar la política exterior de Argentina hacia Tailandia, con principal énfasis en el período 2003-2015, tomando como variables centrales para comprender esta vinculación la relación con la orientación económica interna, y también con variables de corte político-institucional e ideológico que tuvieron, hacia finales del período, un mayor peso en el devenir de las relaciones bilaterales. El recorte temporal tiene como punto de inicio el período de despegue de las exportaciones argentinas hacia Tailandia -de la mano de condiciones internas e internacionales que lo favorecieron- y la redefinición de los puntales de la política exterior argentina. En el recorrido del texto observaremos que, a pesar de comprender los doce años de gobierno del mismo partido -el Frente para la Victoria-, la política exterior hacia Tailandia atravesó períodos de mayor actividad y acercamiento, y de enfriamiento.

Esto nos permite hipotetizar que aunque los objetivos económicos internos proponen el marco y los objetivos nacionales en función de los cuales se implementa determinada orientación de política exterior de manera estructural, en la relación con un Estado individual determinado también influyen de manera determinante demás condiciones internas -y en algunos casos externas-, provocando que dentro de un mismo contexto político y económico se puedan observar diferentes intensidades en los vínculos bilaterales con el mismo socio. A esta hipótesis debe sumarse una variable adicional, que es la prioridad económico-estratégica del socio. En el caso de países prioritarios en la agenda -como Brasil en el caso argentino- la incidencia de variables extraeconómicas es menor. Por el contrario, en el caso de Estados de menor relevancia económica para la inserción del país, las variables extraeconómicas pueden tener mayor incidencia en la relación bilateral, considerando que existe un menor nivel de dependencia del comercio exterior con dicho Estado, lo que repercute en una menor sensibilidad a las alteraciones en la dimensión comercial de la relación.

La política exterior de Argentina hacia Tailandia del período bajo estudio se inscribe de lleno en estas concepciones y permite observar que la consistencia de la política exterior debe comprenderse no como un todo, sino a través del análisis en estratos entre los cuales, dentro de una lógica estructural de la política externa -que se determina en función del modelo económico nacional-, encontramos niveles de menor alcance -relaciones bilaterales con Estados no prioritarios, entre otros-, donde variables menores, más específicas y extra económicas tienen mayor incidencia en la definición de la política exterior. Así, podemos adelantar que en un período que se caracterizó por una búsqueda de diversificación de mercados de exportación -con una clara orientación hacia Asia Oriental- la política exterior hacia Tailandia estuvo sujeta a las condiciones políticas internas del país y a la centralidad 
dada por la Cancillería argentina a la política de derechos humanos e institucionalidad democrática.

II. Los vínculos políticos, sus condicionantes y su relación con las prioridades de política exterior

A modo introductorio, se deben tener en cuenta los antecedentes de las relaciones entre nuestro país y Bangkok a los efectos de entender la evolución de la política exterior argentina y principalmente las acciones llevadas a cabo en los últimos años. Las relaciones diplomáticas se establecieron a través del intercambio de notas efectuado en 1955 por los Embajadores ante los Estados Unidos Hipólito Paz (de Argentina) y Pote Sarasin (por Tailandia) en la ciudad de Washington. En aquellos momentos, la representación diplomática argentina en Bangkok fue la primera de un país latinoamericano en el Sudeste de Asia. Un poco más adelante en el tiempo, para el año 1960, sendas representaciones diplomáticas se convirtieron en embajadas (Embajada Argentina en Tailandia, 2014).

En relación con lo anterior, fue el Presidente Arturo Frondizi (19581962) quien realizó la primera visita oficial al Reino. Esta visita se encontraba en el marco de sus acciones para acercarse a los países asiáticos con el fin de diversificar los mercados de exportación de productos argentinos. Esta visita era parte de una gira alrededor del mundo llevada a cabo por el Presidente, enfocada principalmente en los países asiáticos en vistas de las potencialidades de sus mercados (El Tiempo, 1961). Entre los mencionados acuerdos se destaca el Acuerdo Comercial (ratificado en 1961) para promover las relaciones entre ambos países, en el cual se establecía que harían todos los esfuerzos posibles para lograr el máximo nivel de volumen comercial. A través de este acuerdo se otorgan mutuamente un trato no discriminatorio para sus productos (Ministerio de Relaciones Exteriores y Culto, 1961) ${ }^{2}$.

Para seguir con los antecedentes, recién en 1991 se firmó un acuerdo de cooperación en ciencia y tecnología. Además, en 1996 se ratificó un acuerdo sobre el uso pacífico de la energía nuclear, teniendo en cuenta los intereses por parte de Tailandia en el conocimiento y alto desarrollo sobre energía nuclear alcanzado en Argentina. En 1997 se firmó un acuerdo sobre cooperación en materia de control de estupefacientes y sustancias psicotrópicas. Para comienzos del siglo XXI se rubricó, en el año 2000, un acuerdo de Protección y promoción recíproca de inversiones, en la búsqueda de fomentar la cooperación económica entre ambas naciones (MREC, 2014).

Continuando con las acciones de política exterior ${ }^{3}$ es menester mencionar las distintas visitas oficiales realizadas por ambos países. Por parte de

2. En adelante, nos referiremos por su siglas MREC.

3. Cuando nos referimos a acciones de política exterior, las entendemos como el 
Bangkok, la primera visita oficial fue realizada por el Ministro de Relaciones Exteriores Sit Sawetsila, el 19 de octubre de 1981. La siguiente fue efectuada por el Ministro de Relaciones Exteriores, Prasong Sunsiri, en enero de 1994. Esta visita fue seguida por el Ministro de Relaciones Exteriores Kasemsamosorn Kasemsri en 1996. Desde Argentina, el canciller Di Tella visitó el Reino en junio de 1996. Posteriormente, el Presidente Carlos S. Menem fue recibido por el Rey Bhumiphol y el Primer Ministro Yongjaiyut.

Ya en el marco del período bajo estudio, durante el Gobierno de Néstor Kirchner (2003-2007), precisamente en 2006, se firmó un importante acuerdo de cooperación cultural entre ambas naciones a los fines de fomentar y promover la cooperación en las áreas de artes y cultura; educación e investigación; medios de comunicación; deportes y otras actividades relacionadas con la cultura. Así también, se realizó un memorándum de entendimiento sobre consultas políticas y otras cuestiones de interés común entre los Ministerios de Relaciones Exteriores de sendos países, por el cual se comprometieron a realizar consultas a través de visitas oficiales para intercambiar puntos de vista acerca de temas internacionales de interés mutuo (Rubiolo y Baroni, 2013).

En cuanto a las visitas oficiales de alto nivel, en 2005 se realizó la del Ministro de Relaciones Exteriores Kantathi Suphamongkorn para la celebración de los 50 años de relaciones diplomáticas (Embajada Argentina en Tailandia, 2014).

En el año 2008 se realizó una visita comercial multisectorial a Tailandia dirigida por oficiales del Ministerio de Relaciones Exteriores y acompañada por diferentes empresarios en búsqueda de incrementar el comercio entre ambos países. En octubre de este año se realizó una visita por parte del Viceministro de Relaciones exteriores argentino a Bangkok, tenía como fin participar de la Primera reunión bilateral de consulta bajo el marco del «memorándum de entendimiento sobre consultas políticas y otras cuestiones de interés» firmado en 2006. Además, se buscaba revisar la relación bilateral y analizar las potencialidades de un acercamiento entre MERCOSUR y ASEAN (Rubiolo y Baroni, 2013).

En relación a las reuniones anteriores, en el año 2009 se firmó un memorándum de entendimiento para el programa de asociación para la cooperación conjunta, el primer acuerdo de cooperación triangular que la Argentina firma con un país del Sudeste de Asia (Rubiolo y Baroni, 2013). A partir de este acuerdo, los dos países se comprometen a prestar asistencia

resultado concreto y visible de la acción estatal. El material de la dimensión de las acciones es aquel eminentemente empírico, que puede ser singularizado, situado y datado; es aquel que puede ser metodológicamente objetivado (Lechini, 2006: 23). Las acciones constituyen la implementación de la política exterior. 
técnica conjunta a países en vías de desarrollo mediante un uso efectivo de sus recursos técnicos, humanos y financieros.

De esta forma, en 2010 se lanzó la primera reunión del comité de planificación de cooperación triangular. Además, se pusieron en marcha proyectos de asistencia técnica en materia de desarrollo rural; energía e industria; y ciencia y tecnología. Ya en 2011 se llevó a cabo el primer programa de intercambio sobre la administración de la cooperación Sur-Sur. En esa oportunidad, oficiales de la Agencia Tailandesa de Cooperación y del Ministerio de Relaciones Exteriores fueron capacitados en la administración de la cooperación internacional (Rubiolo y Baroni, 2013). Es pertinente agregar que en diciembre de 2011, funcionarios tailandeses del Centro Nacional de Ingeniería Genética y Biotecnología (National Center of Genetic Engineering and Biotechnology) hicieron una visita a Argentina en el marco de la primera etapa de un proyecto de capacitación en el uso de las ribointerferencia (RNAi). De forma básica, estas técnicas son un potente mecanismo que permite impedir que parte de la información genética que tiene una célula pase a otra nueva (MREC, 2012a). Con la realización de este proyecto, Tailandia busca mejorar desde la Biotecnología el estado de la agricultura y llevar nuevas formas de cultivo a sus productores nacionales. Es decir, actividades importantes para el sector alimenticio de los dos países.

En junio de 2012, tres expertos del equipo argentino de antropología forense viajaron a Bangkok para dar comienzo a un proyecto llamado «Desarrollo de procesos para la Identificación humana». Los tres científicos trabajan en el Instituto Central de Ciencias Forenses (CIFS por sus siglas en inglés) de Tailandia, buscando desarrollar las capacidades de los funcionarios tailandeses en la identificación de restos humanos (MREC, 2012b). En resumen, mediante esta herramienta de cooperación, Bangkok aspira a que sus funcionarios judiciales se capaciten, mientras que los científicos argentinos pueden adquirir nuevos conocimientos y experiencias. Continuando con acciones en el marco de la cooperación Sur-Sur, en julio de 2013 una experta del INTA (Instituto Nacional de Tecnología Agropecuaria) viajó a Tailandia en el marco de un proyecto orientado a potenciar el proceso de selección y mejoramiento genético en Apicultura. La mencionada experta disertó y visitó distintos establecimientos dedicados a la actividad en distintas regiones de Tailandia (MREC, 2013a).

En marzo de 2013, funcionarios de ambos países aprobaron un Plan de Acción 2013-2015 consistente en 15 proyectos de cooperación Sur-Sur, que serán financiados tanto por el Fondo Argentino de Cooperación Sur-Sur y triangular (FO.AR) y la Agencia Tailandesa de Cooperación Internacional. Estos proyectos tienen como objetivo contribuir a las capacidades de los países socios de Argentina de acuerdo con sus propias estrategias de desarrollo (MREC, 2013b). 
En la actualidad se encuentran en funcionamiento ocho proyectos de cooperación técnica entre Argentina y Tailandia en el marco del FO.Ar. De esta manera, la cooperación Sur Sur (CSS) -aunque aún incipiente- se está constituyendo en un eje relevante de la relación bilateral y contribuye al intercambio de experiencias, de conocimientos y de tecnologías al tiempo que favorece el acercamiento entre ambos Estados.

La cooperación técnica es uno de los tipos de la cooperación Sur-Sur. Esta última, aunque tiene sus antecedentes en la década de 1970 y tiene una larga trayectoria en las vinculaciones externas de la Argentina -particularmente con América Latina-, ha sido escasamente implementada en las relaciones con socios asiáticos. La CSS puede ser definida como:

[...] una cooperación política que apunta a reforzar las relaciones bilaterales y/o a formar coaliciones en los foros multilaterales, para obtener un mayor poder de negociación conjunto, en defensa de sus intereses. Se basa en el supuesto que es posible crear una conciencia cooperativa que les permita a los países del Sur reforzar su capacidad de negociación con el Norte, a través de la adquisición de mayores márgenes de maniobra internacional y con ellos, mayor autonomía decisional, para afrontar y resolver los problemas comunes. Es una construcción política que por su propia naturaleza requiere de ciertos supuestos básicos comunes (likemindedness) (Lechini, 2012, p. 18).

En el caso de los ejes prioritarios de la política exterior argentina, la CSS ha sido central en el período bajo estudio. Respecto de los vínculos con Tailandia, esta dimensión de las relaciones está cobrando un mayor dinamismo. La firma de acuerdos previos ha favorecido la implementación y desarrollo de los proyectos, y puede sentar las bases para un crecimiento de este tipo de cooperación en el futuro.

Por último, debemos repasar la relación entre Argentina y Tailandia en ámbitos multilaterales. En esta esfera, el principal espacio de diálogo entre Argentina y Tailandia ha sido el Foro para La Cooperación de América Latina y Asia del Este (FOCALAE). Ambos Estados comparten el interés común de fortalecer la colaboración en el Foro como mecanismo de concertación entre las dos regiones (MREC, 2013c). En esta línea, el Canciller Héctor Timerman participó de la sexta reunión, realizada en Bali, donde se trataron temas relacionados con el comercio entre ambas regiones. Por parte de Argentina, su interés en el Foro tiene que ver con un programa de fortalecimiento de lazos con aquellas regiones que en otros tiempos parecían lejanas. En el marco de esta reunión multilateral, el Canciller mantuvo una reunión con su par tailandés (Ámbito Financiero, 2013). 
Luego de la mencionada cumbre FOCALAE, Timerman la subrayó como el escenario apropiado para, a través de la cooperación Sur-Sur, mejorar la forma en la cual las regiones responden a los desafíos de desarrollo en un mundo en constante cambio. Además, el canciller argentino destacó que los lazos entre ambas regiones se han incrementado en todos los ámbitos. Asimismo, el comercio entre ambas áreas se cuadruplicó en los últimos 10 años, siendo el mayor incremento global entre regiones (MREC, 2013d).

Por su parte, la cancillería argentina lleva adelante un programa destinado a fortalecer los lazos y propiciar las vinculaciones con regiones a las cuales no se consideraba prioridad en décadas anteriores. Cabe destacar que la cooperación Sur-Sur con países de Asia del Este se limitaba en 2003 a dos países y dos proyectos, y actualmente existen 19 proyectos en ejecución. Argentina cree en la relevancia de FOCALAE dada las posibilidades de intercambio de tecnología entre sus miembros y la complementariedad económica (MREC, 2013e).

Teniendo en cuenta todo lo anterior, se puede concluir que los lazos políticos entre Argentina y Tailandia parecerían responder a una estrategia de fortalecimiento de la cooperación Sur-Sur que les permita superar limitaciones del sistema internacional, fomentar el acercamiento entre regiones distantes e incrementar su comercio bilateral como herramientas hacia un mayor nivel de desarrollo. Esto se ha traducido, en el plano de la cooperación, en una elevada cooperación horizontal en el ámbito científico-tecnológico en relación con la mantenida con los demás países del continente asiático-.

No obstante este alentador escenario en la cooperación técnica, los vínculos políticos en su conjunto continúan teniendo una escasa relevancia, debido en parte a la falta de continuidad en la implementación de las acciones de política exterior, la ausencia de visitas oficiales de alto nivel desde Argentina hacia Tailandia desde $1997^{4}$, y las diferencias manifestadas por el gobierno de Tailandia respecto de las medidas de política comercial implementadas por la Argentina (La Nación, 2012; WTO, 2014). A su vez, las condiciones de inestabilidad política en Tailandia desde 2006 -que resultaron en el golpe de Estado del 22 de mayo de 2014- también funcionaron como un obstáculo en el acercamiento político en los últimos años.

A propósito del golpe de Estado, el gobierno argentino manifestó su preocupación a través del Ministerio de Relaciones Exteriores y Culto y realizó «un llamamiento al pleno restablecimiento del orden constitucional y la celebración de elecciones democráticas, al tiempo que exhorta a garantizar el respeto irrestricto de los derechos humanos del pueblo tailandés» (MREC,

4. En febrero de 1997 el Presidente argentino Carlos Menem realizó una gira por el Sudeste de Asia que tuvo como destinos Vietnam, Tailandia y Singapur. 
2014b). De acuerdo a funcionarios de la Embajada Argentina en Bangkok entrevistados por la autora en junio de 2015, la posición negativa del gobierno argentino frente a la continuación del gobierno de facto en Tailandia y al aplazamiento de las elecciones que se realizarían a finales de $2015^{5}$, ha llevado a un enfriamiento de la relaciones bilaterales. Esto se tradujo en la negativa argentina a realizar actos de celebración por el sexagésimo aniversario del establecimiento de los vínculos diplomáticos que se cumplió en febrero de 2015.

A pesar de las manifestaciones, tanto de funcionarios tailandeses como argentinos, del menor dinamismo que se observa en la dimensión política de la relación bilateral, debido principalmente a la postura que el gobierno argentino adoptó frente a los acontecimientos políticos en Tailandia desde 2014, los vínculos comerciales continuaron fortaleciéndose en ese período, incluso frente a las críticas presentadas por el gobierno tailandés a la trabas impuestas por Argentina al comercio exterior. Es decir, mientras se observa un enfriamiento reciente en las relaciones político-diplomáticas, la dimensión económica comercial de la relación mantiene su dinamismo de años anteriores.

En el próximo apartado nos concentramos en las principales características de las relaciones económicas comerciales entre Tailandia y Argentina, y analizaremos el nivel de relevancia de las mismas en el comercio global de nuestro país.

\section{Evolución de las relaciones comerciales}

Los vínculos comerciales de Argentina con el Este de Asia han tenido una tendencia incremental sostenida, particularmente desde 2003 en adelante. Ello se enmarca en una tendencia presente en toda la región latinoamericana de fortalecimiento de las relaciones con Asia, principalmente con China, como socio económico alternativo a Estados Unidos y Europa. La crisis económica de 2008 fortaleció esta tendencia de acercamiento comercial entre América Latina con el Este de Asia (Asian Development Bank, 2012) y demostró a ambas regiones la trascendencia de estos vínculos frente a la depresión económica de sus socios tradicionales.

La región del SEA ha tenido un creciente protagonismo en este fenómeno de dinamización comercial Argentina-Asia. Para el año 2014 el total

5. Las elecciones fueron postergadas varias veces desde que la Junta militar tomó el poder en mayo de 2014. La primera fecha límite fijada para las elecciones había sido octubre de 2015. Ésta fue pospuesta en diciembre de 2014 hacia agosto de 2016, y esta nueva fecha fue recientemente postergada hacia mediados de 2017 (Parameswaran, , 2015). 
comerciado entre Argentina y el Sudeste de Asia ${ }^{6}$ representó el 5,35\% del total comerciado con el mundo, dando cuenta de la relevancia adquirida por estos mercados como socios de nuestro país. Esta relación se ha caracterizado por un sostenido superávit para el comercio local, que en 2014 ascendió a un total de 3.157 millones de dólares. Esta cifra evidencia la relevancia de estos mercados como destinos de las ventas argentinas, cuyo grado de participación en el mismo año ascendió a un 7,54\% de las ventas globales -casi el doble de la participación en 2007, que fue del 3,8\% sobre las exportaciones al mundo-. Esta tendencia puede explicarse por condiciones internas y externas a la Argentina. Entre las primeras encontramos el aumento de la competitividad de los productos argentinos luego de la salida de la convertibilidad en 2002. A ello deben sumarse los cambios introducidos en la producción agrícola, particularmente de semillas, que permitió aumentar la oferta exportadora. En cuanto a las condiciones externas, deben subrayarse los aumentos del precio internacional de los commodities $^{7}$ (CEPAL, 2012) y el incremento de la demanda asiática de productos elaborados por la Argentina que se utilizan como insumos para la producción (principalmente en la cría porcina y avícola).

Dentro de la región, los principales socios comerciales de la Argentina en los últimos años (2007-2014) fueron Indonesia, Tailandia y Malasia teniendo en cuenta el comercio total (ALADI, 2014). Sin embargo, en 2014, el intercambio con Tailandia muestra una leve caída, que desde la perspectiva regional relegó al mercado tailandés al tercer lugar como socio comercial de la Argentina luego de Vietnam e Indonesia. Dado que las exportaciones argentinas a la región del SEA tienen una composición muy similar, y el comercio total en montos con la región se mantuvo casi idéntico entre 2013 y 2014, observamos que la caída de los montos intercambiados con Tailandia -principalmente las exportaciones argentinas-, es proporcional al incremento que se observa en las exportaciones a Vietnam en el mismo año.

6. Tomando como referencia los principales seis socios comerciales de la región: Filipinas, Indonesia, Malasia, Singapur, Tailandia y Vietnam.

7. De acuerdo al informe de la CEPAL, entre 2000 y 2008, la tasa media de crecimiento del precio de las exportaciones de América Latina fue del 7,6\%, en tanto que la del volumen fue de solo el $4,2 \%$. Los productos cuyos precios tuvieron las mayores tasas de crecimiento fueron el petróleo crudo, el cobre, el mineral de hierro, las semillas de soja y el gas natural, entre otros (CEPAL, 2012: 76). 


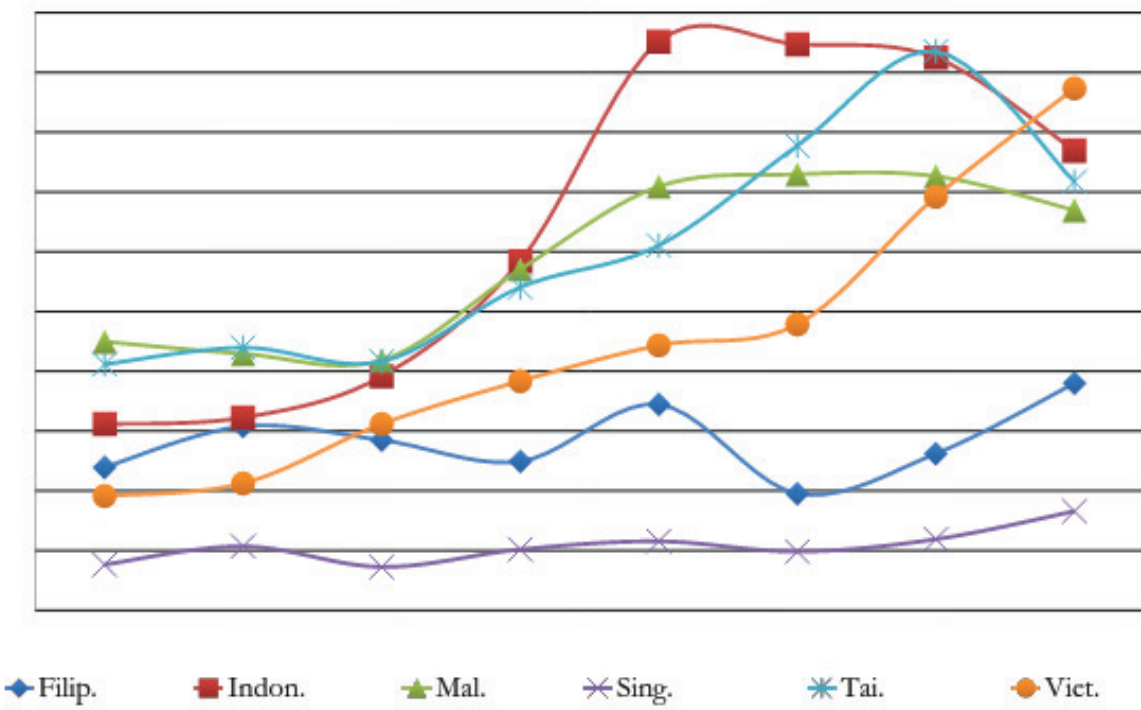

Fuente: elaboración propia con datos de ALADI (2015).

Los vínculos comerciales tuvieron una evolución positiva desde 2002 en adelante. Entre ese año y 2004 encontramos un crecimiento en las exportaciones argentinas hacia Tailandia del 20\% (ALADI, 2014). Entre los años 2002 y 2007 se puede observar un aumento del comercio bilateral, que hasta 2004 arrojó un saldo favorable a la Argentina, tendencia que se revirtió en 2005 y se mantuvo hasta 2014 -con la única excepción del año 2009- (Gráfico II). Es necesario explicitar que las exportaciones argentinas tuvieron un alto grado de concentración en aquellos productos derivados del complejo oleaginosos sojero destinados a la alimentación animal. Es decir que dependió durante esos años de la evolución de la industria alimentaria tailandesa. Por este motivo, durante la época de la gripe aviar que afectó a las exportaciones tailandesas de pollos, principalmente en 2004, se vieron disminuidas las importaciones de alimento para animales desde Argentina ${ }^{8}$.

8. Los brotes de gripe aviar en 2003-2004 dieron lugar a una disminución sin precedentes del $8 \%$ del comercio mundial de carne de aves en 2004 y generaron en un primer momento un alza de $20 \%$ a $30 \%$ en el precio internacional de la carne de aves. Tailandia es un importante abastecedor mundial de este tipo de carnes y vio sus exportaciones frenadas por distintos embargos sanitarios. A su vez, el continente asiático ha sido uno de los más afectados por este brote y ha sufrido un impacto económico negativo debido a que el comercio avícola tiene un rol importante en sus economías, llegando en el caso de Tailandia a representar el 0,5\% del PBI.

Para el brote de 2004, las exportaciones tailandesas se redujeron en un $41 \%$ en términos monetarios, y en términos de volumen en un $63 \%$ en comparación con 
La harina y los porotos de soja representaron el $75 \%$ de las ventas argentinas en este período, seguidas por los cueros, con un $7 \%$; es decir que no hay una diversificación en la canasta exportadora.

Por su parte, desde Tailandia (siguiendo la clasificación por grupo de ítems arancelarios de ALADI) las importaciones se compusieron de reactores nucleares, calderas, máquinas, aparatos y artefactos mecánicos y sus partes en un 44\%; de automóviles, tractores, velocípedos y demás vehículos terrestres y sus partes en un 13\%; máquinas y aparatos eléctricos y sus partes en un 10\%; caucho y sus manufacturas en un $8 \%$; conservas y preparaciones de carne, pescados, crustáceos y moluscos en un 5\%; así como manufacturas de fundición de hierro o acero en un 5\% (ALADI, 2014). Estos grupos de ítems representan el 85\% de las exportaciones tailandesas hacia Argentina. Una composición parecida se observa para el período 2008-2013, lo que permite afirmar que estructuralmente el país asiático tiene una canasta de exportación diversificada y basada en bienes con mayor grado de valor.

\section{Gráfico II. Comercio Argentina-Tailandia, 2002-2014}

En miles de dólares

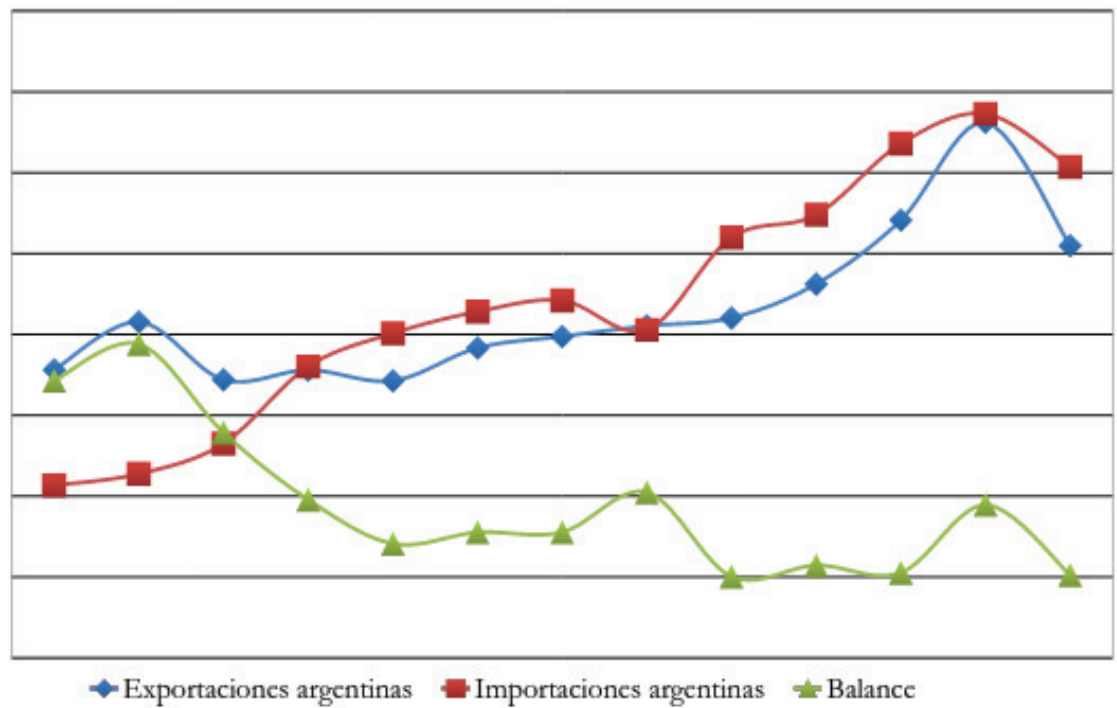

Fuente: elaboración propia con datos de ALADI (2015).

A continuación se analizará con mayor profundidad el comercio entre Argentina y Tailandia entre los años 2007 y 2014. En el Gráfico II se observa que durante el período bajo estudio se han incrementado paulatinamente 
los intercambios entre Argentina y Tailandia. Tanto las exportaciones como las importaciones ascendieron desde los 285 y 403 millones de dólares respectivamente en 2007 hasta los 619,6 y 814,5 millones de dólares en 2014. Tailandia es el principal mercado de origen de las importaciones argentinas en el Sudeste de Asia desde 2005. Este rol se acentuó en 2010, y se mantiene hasta 2014, siendo Malasia el segundo mercado de origen con un monto importado por Argentina de 398 millones de dólares, menos de la mitad de lo comprado a Tailandia el mismo año.

En relación con este rol de Tailandia como proveedora de productos al mercado local, se mantuvo el déficit comercial para la Argentina -en contraste con el superávit que nuestro país tuvo con la región del SEA durante 2007-2014-. El déficit con Tailandia -que se mantuvo en un promedio anual de 190 millones de dólares entre 2010 y 2012- se redujo drásticamente en 2013 a 21,4 millones, pero en 2014 volvió a dispararse a 194 millones. Hubo un aumento exponencial de la venta de pellets y harina de soja entre 2012 y 2013. Las mismas pasaron de 261,9 millones de dólares en 2011 a 497,6 en 2012 y 658 millones en 2013. Este fenómeno resultó en un aumento de las ventas argentinas al mercado tailandés de un $76 \%$ en dos años, mayormente explicado por el boom de las ventas de residuos de la industria oleaginosa para la alimentación animal. Entretanto, las importaciones argentinas desde el socio asiático aumentaron un $36 \%$ en el mismo período.

Desde el punto de vista de la composición del comercio, observamos en el período una alta concentración de la canasta exportadora argentina en escasos productos de origen agropecuario y, por otro lado, un alto grado de diversificación en la canasta importadora con productos de origen industrial.

Tomando como punto de análisis el año 2012 se puede observar que consisten principalmente en harina y pellets de soja con un $73 \%$ de participación, seguido por cueros y pieles de bovino con un $8 \%$ y porotos de soja con un $4 \%$ de participación. La composición se completa con autopartes y otras pieles y cueros, con un $3 \%$ y $2 \%$ respectivamente (Gráfico III). La principal conclusión que se obtiene del análisis es que el complejo sojero es responsable del $77 \%$ de las exportaciones totales argentinas a Tailandia y que hay una concentración de un $85 \%$ en manufacturas de origen agropecuario para el año 2012. En 2013 se reprodujo un patrón similar, dado que el 71\% de las ventas se compuso de harina y pellets de soja, el $8 \%$ de porotos de soja, y el $8 \%$ de cueros y pieles. Estos tres productos dieron cuenta del $87 \%$ del total exportado.

Si se tienen en cuenta las importaciones provenientes de Tailandia para el año 2012, se puede observar en el Gráfico III que están concentradas en Manufacturas de Origen Industrial (MOI), principalmente autopartes, con un 22\%. Le siguen en importancia las partes destinadas a motores, con un $7 \%$, y las máquinas para el procesamiento de datos, con un 6\%. Por último 
se encuentran las bombas de aire o vacío, con un 5\%, y las bombas para líquidos, con un 4\% de participación. Le siguen con una importancia relativa menor, productos de goma; conservas de pescado y crustáceos, productos de acero y hierro, máquinas de aire acondicionado y polímeros de etileno, propileno y otros en su forma primaria. Se observa una canasta de importación diversificada y con participación de MOI que participan de cadenas de valor como lo son las autopartes.

\section{Gráfico III. Composición del comercio bilateral Argentina-Tailandia. Año 2012}
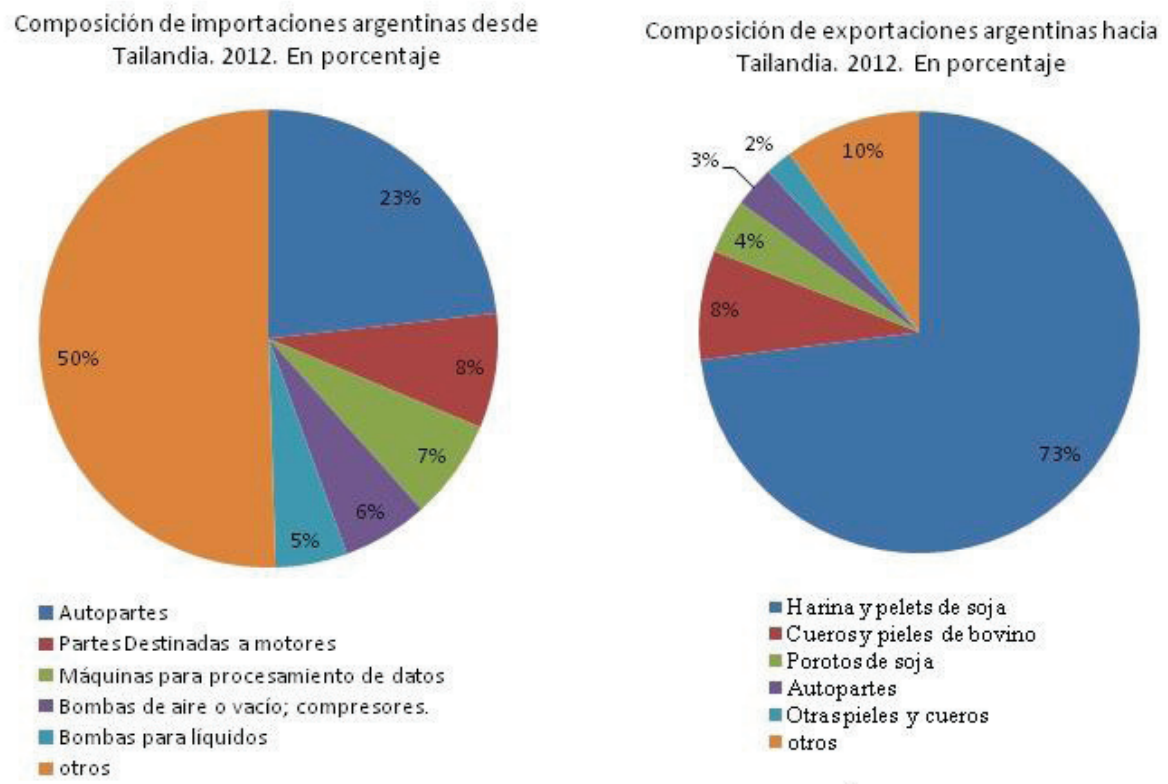

Fuente: elaboración propia con datos de la Dirección Nacional de Estrategia de Inversiones y Promoción Comercial (2013).

En lo referente a las relaciones diplomáticas bilaterales en materia económica, desde el año 2004 funcionarios de Argentina y de Tailandia mantienen reuniones bilaterales de consulta. En la segunda reunión bilateral, llevada a cabo en 2008, se abarcó una gran variedad de temas y las partes reafirmaron su voluntad de trabajo conjunto para afianzar esta relación que consideran estratégica. Se acordó continuar en la promoción de inversiones y comercio bilateral; intentar complementar los puntos fuertes de cada país, es decir Tailandia como puerta de entrada al Sudeste Asiático y Argentina como enlace hacia el MERCOSUR. También se comprometieron a establecer acuerdos de cooperación en materia aduanera, en temas pesqueros y agrícolas, además de comprometerse en la cooperación en el área energética, 
especialmente el gas natural comprimido para vehículos (Embajada de Tailandia en Argentina, 2011).

A modo de recapitulación, es en la dimensión comercial donde se manifiesta el mayor dinamismo e interés de la Argentina en las relaciones bilaterales con Tailandia. Los cambios en los flujos de intercambio están generando un nuevo escenario comercial que se presenta más favorable a la Argentina en términos de saldos de comercio. Sin embargo, este fenómeno se está desarrollando en paralelo a -y como resultado de- una mayor concentración de la canasta exportadora en materias primas y MOA, reproduciendo el patrón de reprimarización que se evidencia en las relaciones comerciales de Argentina con la región del SEA y con el Este de Asia en su conjunto.

\section{Reflexiones finales}

El punto neurálgico de este trabajo ha sido, además de realizar una revisión actualizada de las relaciones en las dimensiones política y económica de Argentina con Tailandia, sentar algunas bases teóricas para el análisis de la política exterior de nuestro país con otros Estados en vías de desarrollo o del Sur. Como planteamos en la Introducción, la política exterior de un país en desarrollo refleja -primordialmente, aunque no únicamente- los lineamientos y objetivos del modelo económico interno. De esta manera, debe subrayarse que las relaciones Argentina-Tailandia se encontraron enmarcadas, entre 2003-2015, en la égida de una política exterior orientada a la diversificación de socios comerciales que permitiera aumentar los márgenes de autonomía del país, disminuyendo la dependencia sobre grandes socios, emergentes o tradicionales. La crisis de 2008, que fuertemente afectó a dichos Estados del hemisferio boreal, y que tuvo menores repercusiones en la región del Sudeste Asiático, alentó el ya comenzado acercamiento de la Argentina a los Estados de dicha región.

Históricamente, la relación con Tailandia ha tenido escasa relevancia en la agenda externa de la Argentina; sin embargo desde 2003 en adelante, en el marco de una estrategia de diversificación, los lazos comerciales con este país han tomado mayor dinamismo. A este respecto, la similitud económica (haciendo referencia al posicionamiento y tamaño de ambas) ha resultado de extrema ventaja a la hora de relacionar a ambos Estados. No obstante, en el ámbito económico-comercial particularmente, si bien los dos países se han posicionado como socios comerciales importantes para cada una de las partes, sumado al hecho que la relación se encuadra en la lógica de las vinculaciones Sur-Sur, las relaciones entre estos Estados, a pesar de los esfuerzos por lograr la incorporación de ambas economías a cadenas de valor agregado, todavía parecen reproducir patrones de vinculación Norte-Sur en 
el sentido de que la Argentina aún exporta en su mayoría productos de bajo/ nulo valor agregado (materias primas) a cambio de manufacturas de origen industrial. En este sentido, la diversificación productiva se ha visto ralentizada por esta situación, a pesar de que sí se evidencia una diversificación de socios.

En la dimensión político-diplomática, la relación con el Reino de Tailandia ha sido constante hasta el año 2014 por los motivos que mencionamos arriba. Sin embargo, el interés de ambas no se presenta simétrico, principalmente porque para la Argentina los vínculos con el Sudeste de Asia han girado más en torno a las relaciones con Indonesia y con Malasia, y recientemente Vietnam. Este fenómeno se ha visto aún más profundizado con el enfriamiento diplomático que se originó desde el golpe de Estado en Tailandia y el posicionamiento del gobierno argentino al respecto. Esta variable interna -la reinvindicación de los derechos humanos y la institucionalidad democrática - que afecta la política exterior argentina hacia Bangkok también pudo haber sido una de las variables que incidieron en la disminución del comercio bilateral, así como en la preferencia por fortalecer vínculos económicos con otros socios de la región. Más allá del cambio de gobierno en la Argentina - por el cual el triunfo de la Alianza Cambiemos, encabezada por Mauricio Macri, puso fin a 12 años de gobierno del Frente para la Victoria- el mantenimiento de una situación de inestabilidad y de un gobierno de facto en Tailandia puede continuar resultando un obstáculo a los vínculos bilaterales, si se mantienen -como es de prever- los principios de reivindicación democráticos y de DDHH en política exterior desde la Cancillería nacional.

En cuanto a la cooperación bilateral, existe una amplia gama de acuerdos firmados en numerosos campos por fuera de las fronteras económicocomerciales. Este rasgo excepcional de los vínculos que Argentina mantiene con Tailandia puede redundar no solo en una transferencia de conocimiento y tecnologías, sino también en un mayor conocimiento mutuo que se derrame hacia otras áreas de la relación.

Finalmente, la dimensión cultural -que se constituye en un eje central para el fortalecimiento de los lazos entre ambos Estados- es la más débil y continúa siendo uno de los obstáculos en el fortalecimiento de las vinculaciones. No obstante las relaciones con el Sudeste Asiático se han planteado como una estrategia en la búsqueda de mayor autonomía y disociación de los históricos socios comerciales argentinos, la distancia cultural con aquella región dificulta el desarrollo e institucionalización de los lazos Argentina-SEA. 


\section{Bibliografía}

ALADI (2015). Sistema de información de Comercio Exterior. (ALADI:

Montevideo) (on line) (Consulta: 04/02/2015), http://consultawebv2.aladi. org/sicoexV2/jsf/home.seam.

Ámbito Financiero (2013). Visita oficial para incrementar las ventas a Asia. (Buenos Aires) (on line) (Consulta: 07/o7/2014), http://www.ambito.com/diario/ noticia.asp?id=692801

Asian Development Bank. Shaping the future of the Asia and the Pacific - Latin America and the Caribbean relationship. (Asian Development Bank, InterAmerican Development Bank, and Asian Development Bank Institute: Manila, 2012) (on line) (Consulta: 12/o9/2014), http://idbdocs.iadb.org/ wsdocs/getdocument.aspx?docnum $=36836575$

Busso, Anabella (2014). Los vaivenes de la política exterior argentina redemocratizada (1983-2013). Reflexiones sobre el impacto de los condicionantes internos, en Estudios Internacionales, n. 177, pp. 9-33.

Dirección Nacional de Estrategia de Inversiones y Promoción Comercial (2013). Informes por país. (Buenos Aires: MRECIC) (on line) (Consulta: 22/o8/2014), http://www.inversiones.gov.ar/userfiles/tailandia.pdf.

El Tiempo (1961). Viaje alrededor del Mundo Inicia hoy Arturo Frondizi. (Bogotá, 1961) (on line) (Consulta: 02/07/2014), http://news.google.com/newspap ers?nid $=1706 \&$ dat $=19611127 \&$ id $=$ scAqAAAAIBAJ $\&$ sjid $=82$ gEAAAAIBAJ $\&$ $\mathrm{pg}=4786,652488$

Embajada Argentina en Tailandia (2014). Reseña Histórica y Acuerdos Firmados. (Bangkok) (on line) (Consulta: 02/06/2014), http://www.etail.mrecic.gov. ar/node/4221

Embajada de Tailandia en Argentina (2011). Tailandia y Argentina. 50 años de Relación. (Buenos Aires) (on line) (Consulta: 02/07/2014), http://www. thaiembargen.org/thaiarg.php?id_seccion $=0$

La NACión (2012). "Dura queja de 40 países por las trabas a las importaciones". 31 de marzo. (on line). (Consulta: 14/o9/2014), http://www.lanacion.com. ar/1461287-dura-queja-de-40-paises-por-las-trabas-a-las-importaciones.

LeCHINI, Gladys (2009). La Cooperación Sur-sur y la búsqueda de autonomía en América Latina: ¿Mito o realidad?. Relaciones internacionales, 12: 55-80.

Lechini, Gladys. (2012). Reflexiones en torno a la Cooperación Sur-Sur. En Morasso, C. y Pereyra Doval, G. (comp); Argentina y Brasil: "Proyecciones Internacionales, Cooperación Sur-Sur e Integración”. (Rosario: UNR Editora): 13-26.

Ministry of Foreign Affaris of the Kingdom of Thailand (2013). "The inauguration of Thailand Corner at Catholic University of Salta”. (Bangkok) (on 
line) (Consulta: 06/10/2014), http://www.mfa.go.th/main/en/ media-center/30/39762-The-inauguration-of-\%E2\%80\%9CThailandCorner\%E2\%80\%9D-at-Catholic.html.

MREC (1961). Acuerdo de comercio entre el gobierno de la República Argentina y el gobierno del Reino de Tailandia. (Consulta: 01/07/2014), http://tratados. cancilleria.gob.ar/busqueda.php?consulta $=$ si\&modo $=\mathrm{c}$

MREC (2006). Acuerdo de cooperación cultural entre el gobierno de la República Argentina y el gobierno del Reino de Tailandia. (Consulta: 23/06/2014), http://tratados.cancilleria.gob.ar/busqueda.php?consulta $=$ si\&modo $=\mathrm{c}$

MREC. (2012a). Tailandia: Capacitación para el uso de biotecnología. (Consulta: 01/07/2014), http://www.mrecic.gov.ar/es/ tailandia-capacitacion-para-el-uso-de-biotecnologia

MREC (2012b). Tailandia: Desarrollo de Procesos para la Identificación Humana. (Consulta: 02/07/2014), http://www.mrecic.gov.ar/es/ tailandia-desarrollo-de-procesos-para-la-identificaci\% $\mathrm{C}_{3} \%$ B3n-humana

MREC (2013a). Tailandia: Producción apícola, productos y mercados. (Consulta: 02/o6/2014), http://www.mrecic.gov.ar/ tailandia-produccion-apicola-productos-y-mercados.

MREC (2013b). Argentina y Tailandia triplican su cooperación Sur-sur. Buenos Aires (Consulta: 02/06/2014), http://www.mrecic.gov.ar/es/ argentina-y-tailandia-triplican-su-cooperacion-sur-sur-o

MREC (2013c). Argentina - Tailandia: Reunión de Consultas Políticas en Buenos Aires. (Consulta: 02/06/2014), http://www.mrecic.gov.ar/ argentina-tailandia-reunion-de-consultas-politicas-en-buenos-aires

MREC (2013d). Concluyó la VI Reunión Ministerial del Foro de América Latina y Asia del Este (FOCALAE). (Consulta: 10/o6/2014), https://www.mrecic. gov.ar/concluyo-la-vi-reunion-ministerial-del-foro-de-america-latina-y-asiadel-este-focalae

MREC (2013e). Timerman viaja a Indonesia para participar del Foro de América Latina y Asia del Este. (Consulta: 10/o6/2014), https://www.mrecic.gov.ar/ timerman-viaja-indonesia-para-participar-en-el-foro-de-america-latina-yasia-del-este.

MREC (2014a). Biblioteca digital de tratados. (Consulta: 25/o6/2014), http:// tratados.mrecic.gov.ar/busqueda.php.

MREC (2014b). El Gobierno argentino expresa su preocupación por la ruptura del orden institucional en Tailandia. (Consulta: 03/02/2016), http://www. mrecic.gov.ar/el-gobierno-argentino-expresa-su-preocupacion-por-laruptura-del-orden-institucional-en-tailandia.

Prashanth Parameswaran (2014). No Thai Election Until At Least February 2016: Deputy Prime Minister, en The Diplomat, diciembre. 
Consuta: 04 febrero 2016. http://thediplomat.com/2014/12/

no-thai-election-until-at-least-february-2016-deputy-prime-minister/

Prashanth Parameswaran (2015). When Will Thailand's Elusive Election Be Held?, en The Diplomat, septiembre. Consuta: 04 febrero 2016. http://thediplomat. com/2015/o9/when-will-thailands-elusive-election-be-held/

Rosales, Osvaldo; Kuwayama, Mikio (2012). China y América Latina y el Caribe. Hacia una relación comercial estratégica. (on line) CEPAL: Santiago de Chile. (Consulta: julio 2014), http://www.cepal.org/publicaciones/xml/9/46259/ China_America_Latina_relacion_economica_comercial.pdf.

Rubiolo, M. Florencia y BAroni, Paola (2013). South-South Bilateral Relations: Argentina's Political and Trade Links with South-east Asia, Jurnal global dan strategis, Centre for Strategic and Global Studies, Airlangga University, 7(1), Surabaya, Indonesia: pp. 1-16.

Rubiolo, M. Florencia; Morero, Hernán y Santillán, Gustavo. (2010). La política exterior argentina hacia el sudeste de Asia entre las presidencias de Carlos Menem y Néstor Kirchner desde una mirada constructivista. Portes. Revista mexicana de estudios sobre la Cuenca del Pacífico, 4: 83-107.

Rubiolo, M. Florencia y Ramoneda, Ezequiel (2012). Argentina-Indonesia: creciente interdependencia comercial y revitalización de los vínculos políticos (19992012). Astrolabio, Centro de Investigaciones y Estudios sobre Cultura y Sociedad - CONICET - UNC, 9: 62-86.

Spoor, M. (2010). Asia y la economía mundial «Caminando con dos piernas (desiguales)». Revista CIDOB d'Afers Internacionals, 89-90: 45-62. (on line) (Consulta: 12/09/2014), http://www.raco.cat/index.php/revistacidob/ article/viewFile/191565/257474.

SAlles Almeida, Juliana (2006). Gripe aviar: los impactos comerciales de las barreras sanitarias y los desafíos para América Latina y el Caribe. CEPAL: División comercio internacional e Integración. (on line). (Consulta: 05/o9/2014), http://www.rlc.fao.org/es/prioridades/transfron/aviar/pdf/ Comin.pdf

Simonoff, Alejandro. (2009). Regularidades de la Política Exterior de Néstor Kirchner. Confines de Relaciones Internacionales y Ciencia Política, Tecnológico de Monterrey, 10: 71-86. (on line). (Consulta: 15/03/2014), http://confines.mty.itesm.mx/articulos10/Simonoff1.pdf

VAN Klaveren, Alberto (1992). Entendiendo las políticas exteriores latinoamericanas. Modelo para armar, en Estudios Internacionales, abriljunio, pp: 169-216.

WTO (2014). "Argentina - Measures Affecting the Importation of Goods". September. (on line). (Consulta: 06/10/2014), http://www.wto.org/english/ tratop_e/dispu_e/cases_e/ds438_e.htm. 


